\section{The peril of adjusting for baseline when using change as a predictor}

\author{
Kimmo Sorjonen ${ }^{1}$, Daniel Falkstedt ${ }^{2}$, \\ Bo Melin ${ }^{1}$, \& Michael Ingre ${ }^{1,3,4}$
}

\footnotetext{
${ }^{1}$ Department of Clinical Neuroscience, Karolinska Institutet, Stockholm, Sweden

${ }^{2}$ Institute of Environmental Medicine, Karolinska Institutet, Stockholm, Sweden

3 Department of Psychology, Stockholm University, Stockholm, Sweden

${ }^{4}$ Institute for Globally Distributed Open Research and Education (IGDORE), Stockholm, Sweden
}

Some studies have analyzed the effect of a predictor measured at a later time point (X1), or of the X1-X0 difference, while adjusting for the predictor measured at baseline (X0), on some outcome Y of interest. The present simulation study shows that, if used to analyze the effect of change in $\mathrm{X}$ on $\mathrm{Y}$, there is a high risk for this analysis to produce type 1errors, especially with a strong correlation between true $\mathrm{X}$ and $\mathrm{Y}$, when $\mathrm{X} 0$ and $\mathrm{X} 1$ are not measured with very high reliability, and with a large sample size. These problems are not encountered if analyzing the unadjusted effect of the $\mathrm{X} 1-\mathrm{X} 0$ difference on $\mathrm{Y}$ instead, and as this effect exhibits power on par with the adjusted effect it seems as the preferable method when using change between two measurement points as a predictor.

Key words: Adjusting for baseline; change; power; prediction; regression; reliability; simulation; type 1-error

\section{Introduction}

It has been demonstrated before that it can be problematic to claim an effect of a predictor $X$ on the change in an outcome $\mathrm{Y}$ based on a found effect of $\mathrm{X}$ on $\mathrm{Y}$ at follow up (Y1), alternatively on the change in the outcome from baseline to follow up (Y1-Y0), while adjusting for the outcome at baseline $(\mathrm{Y} 0)$. The problem lies in the fact that if there is an association between $\mathrm{X}$ and the subjects' true value on $\mathrm{Y}$, there will also tend to be an association between $\mathrm{X}$ and degree of measurement error at baseline given the same score on $\mathrm{Y0}$, and as degree of measurement error is predictive, due to regression toward the mean, of change to the next measurement, we will tend to see an effect of $X$ on $\mathrm{Y} 1$, or on $\mathrm{Y} 1-\mathrm{Y} 0$, while adjusting for $\mathrm{Y} 0$, even if no true change has taken place (Eriksson \& Häggström, 2014; Sorjonen, Melin, \& Ingre, 2019).

As an example, imagine that we analyze the effect of anxiety, as measured at baseline, on the change in depression between baseline and followup while adjusting for measured depression at baseline. Some might think that a significant positive effect warrants the conclusion that high degree of anxiety predicts an increase in depression.
However, as there probably is a positive association between true anxiety and true depression, and we do not measure anxiety or depression with perfect reliability, we can assume that among people with the same measured depression at baseline, those with a higher anxiety score are likely to have a higher true depression than those with a lower anxiety score, i.e. there is a negative association between measured anxiety and the error in the measurement of depression given the same measured depression. And as negative measurement error is predictive of an increase while positive error is predictive of a decrease, due to regression toward the mean, we stand a good chance of observing a positive association between measured anxiety at baseline and change in depression between baseline and follow-up if we adjust for measured depression at baseline, even if no true change in depression has taken place.

Although it seems less common, change can also be used as a predictor. Some studies analyze, as recommended by Rosner (1979), the effect of a predictor measured at a later time point (X1), or of the $\mathrm{X} 1-\mathrm{X} 0$ difference, on an outcome $\mathrm{Y}$ while adjusting for the predictor measured at an earlier time point (X0). Studies employing this method claim, for instance, to have demonstrated an effect of change in blood pressure on mortality (Menotti et al., 2004) and on cardiovascular diseases (Parizadeh et al., 2017).

However, using change between two measures as a predictor while adjusting for baseline might be as problematic as using it as outcome. A combination of an association between the true value on the predictor and the outcome and error in the measurement of the predictor might result in an association between observed change in the predictor and the outcome even if there is no association between true change in the predictor and the outcome (Cain, Kronmal, \& Kosinski, 1992). Keeping to the example with anxiety and depression, given the same anxiety score at baseline, those who increase in anxiety to follow-up have probably, on average, higher true anxiety than those who decrease in measured anxiety. And as true anxiety is positively associated with depression, we will probably see a positive association between observed change in anxiety between baseline and follow-up and depression, adjusting for measured anxiety at baseline, even if no true change in anxiety has taken place.

The objective of the present simulation study was to have a closer look at the effect of a predictor measured at a later time point (X1) while adjusting for the predictor measured at an earlier time point (X0), and to compare this effect with the unadjusted effect of the X1-X0 difference, both considering risk for type 1-error and power. 
Method

Using R 3.5.2 (R Core Team, 2018) data was simulated through the following steps (Figure 1, script available at https://osf.io/rjcus/): (1) Virtual subjects were allocated a true $\mathrm{X}$ score from a random normal distribution; (2) The virtual subjects were allocated observed X0 and X1 scores from a random normal distribution with various defined population correlations (same for both) with the true $\mathrm{X}$ score (i.e. square root of reliability); (3) The virtual subjects were allocated an observed Y score from a random normal distribution with various defined population correlations with the true X score.

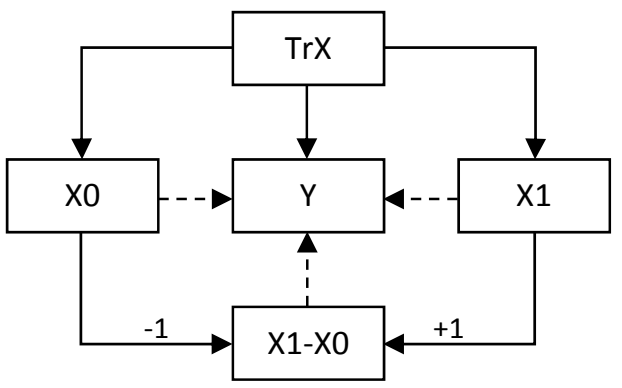

Figure 1. Steps (solid lines) as well as analyzed effects (dashed lines) in the first simulation with no true association between $\mathrm{Y}$ and a change from $\mathrm{X} 0$ to X1.

The adjusted effects of $\mathrm{X} 0$ and $\mathrm{X} 1$, as well as the unadjusted effect of the X1-X0 difference, on Y was analyzed with ordinary least squares regression. The standardized regression effect of $\mathrm{X} 1$ on $\mathrm{Y}$ while adjusting for $\mathrm{X} 0$ is given by the equation (Cohen, Cohen, West, \& Aiken, 2003):

$$
\beta_{X 1, Y . X 0}=\frac{r_{X 1, Y}-r_{X 0, Y} r_{X 0, X 1}}{1-r_{X 0, X 1}^{2}}
$$

(Equation 1)

As the correlations between $\mathrm{X} 0, \mathrm{X} 1$, and $\mathrm{Y}$ in the present simulation equals the product of their correlations with true X (see Figure 1), the expected adjusted effect is given by (see Appendix):

$$
E\left|\beta_{X 1, Y . X 0}\right|=\frac{r_{X 0, Y}+r_{X 1, Y}}{2\left(1+r_{X 0, X 1}\right)}
$$

(Equation 2)

In Equation 2 we see that the effect of $\mathrm{X} 1$ on $\mathrm{Y}$ while adjusting for $\mathrm{X} 0$ is expected to be zero only when $\mathrm{X} 1$ and X0 have equally strong correlations with Y but with opposite signs. The unadjusted effect of the $\mathrm{X} 1-\mathrm{X} 0$ difference on $\mathrm{Y}$, on the other hand, is given by $r_{T r X, Y} \times r_{T r X, X 1}-r_{T r X, Y} \times r_{T r X, X 0}=r_{T r X, Y}\left(r_{T r X, X 1}-\right.$ $r_{\operatorname{Tr} X, X 0}$ ) (see Figure 1), i.e. given that $r_{\operatorname{Tr} X, X 1}=r_{\operatorname{Tr} X, X 0}$ it is always expected to be zero.

A first objective of the present study was to calculate the probability for a significant result, i.e. type 1-error, when analyzing the effect of X1 on Y while adjusting for $\mathrm{X} 0$, as well as the unadjusted effect of the X1-X0 difference on Y, when data was generated as depicted in Figure 1. We also wanted to see how the risk for type 1-error is affected by the strength of the association between true $\mathrm{X}$ and $\mathrm{Y}$ (drawn from a random uniform distribution between 0 and 1 ), the reliability in the measurement of X0/X1 (set to $0.4,0.6,0.8$, or 0.99 (the calculations did not converge if reliability was set to 1)), as well as sample size $(20,100,500$, or 2500$)$. As shown above, differently from the unadjusted effect of the $\mathrm{X} 1-\mathrm{X} 0$ difference, the effect of $\mathrm{X} 1$ on $\mathrm{Y}$ while adjusting for $\mathrm{X} 0$ is usually not expected to be zero, and therefore we predict that this latter analysis is more prone to result in type 1-errors.

In a second set of simulations, we added true change in $\mathrm{X}$ to the model and allowed this true change to influence $Y$ to various degrees. The size of the true change was, in its turn, influenced by the true $\mathrm{X} 0$ score to various degrees (Figure 2). Again, the effect of $\mathrm{X} 1$ on $\mathrm{Y}$ while adjusting for $\mathrm{X} 0$, as well as the unadjusted effect of the X1-X0 difference, were analyzed with ordinary least squares regression. Correlation between true change in $\mathrm{X}$ and $\mathrm{Y}$, reliability in the measurement of $\mathrm{X} 0 / \mathrm{X} 1$, and sample size were varied as described above while the correlation between true $\mathrm{X} 0$ and true change in $\mathrm{X}$ was set to $-0.7,0$, or 0.7 .

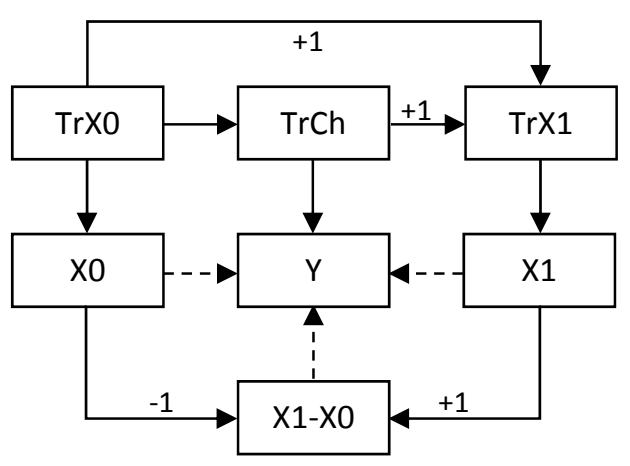

Figure 2. Steps (solid lines) as well as analyzed effects (dashed lines) in the second simulation with a true association between $\mathrm{Y}$ and a change from $\mathrm{X} 0$ to $\mathrm{X} 1$.

\section{Results}

\section{With no true change in $X$}

When generating data as depicted in Figure 1, the risk for type 1-error was always close to the nominal 5\% when analyzing the unadjusted effect of the X1-X0 difference on Y (dashed lines in Figure $3)$. When analyzing the effect of $\mathrm{X} 1$ on $\mathrm{Y}$ while adjusting for $\mathrm{X} 0$, on the other hand, a very high risk for type 1-error could be seen, especially with a strong association between true $\mathrm{X}$ and $\mathrm{Y}$, when $\mathrm{X}$ /X1 were not measured with very high reliability, and with a large sample size (solid lines in Figure 3).

\section{With true change in $X$}

When generating data as depicted in Figure 2, we see the lowest probability for a significant finding, i.e. power, when analyzing the effect of $\mathrm{X} 1$ on $\mathrm{Y}$ 
while adjusting for $\mathrm{X} 0$ in a situation with a negative association between true $\mathrm{X} 0$ and true change in $\mathrm{X}$ (grey dashed lines in Figure 4), especially when $\mathrm{X} 0 / \mathrm{X} 1$ are measured with low reliability and with a small sample size. With no or a positive correlation between true $\mathrm{X} 0$ and true change in $\mathrm{X}$, the effect of $\mathrm{X} 1$ while adjusting for $\mathrm{X} 0$ demonstrated a slight power-advantage over the unadjusted effect of the $\mathrm{X} 1$-X0 difference, but only if X0/X1 were measured with low reliability and the sample size was small (the dotted and solid grey lines are slightly above the corresponding black lines in the upper left panel in Figure 4).
Discussion

The present simulation indicates that a significant effect of a predictor (X1) on an outcome (Y) while adjusting for the predictor measured at an earlier point in time (X0) can often be observed even without any true association between change in $\mathrm{X}$ and $Y$, as long as there is an association between true $\mathrm{X}$ and $\mathrm{Y}$. The probability is accentuated if $\mathrm{X} 0$ and $\mathrm{X} 1$ are not measured with very high reliability and by a large sample size. Unadjusted effects of the X1$\mathrm{X} 0$ difference do not exhibit the same tendency to produce type 1-errors and as this latter effect does not seem to suffer from any general or excessive shortage of power compared to the effect of X1 while adjusting $\mathrm{X} 0$, in some situations it even seems

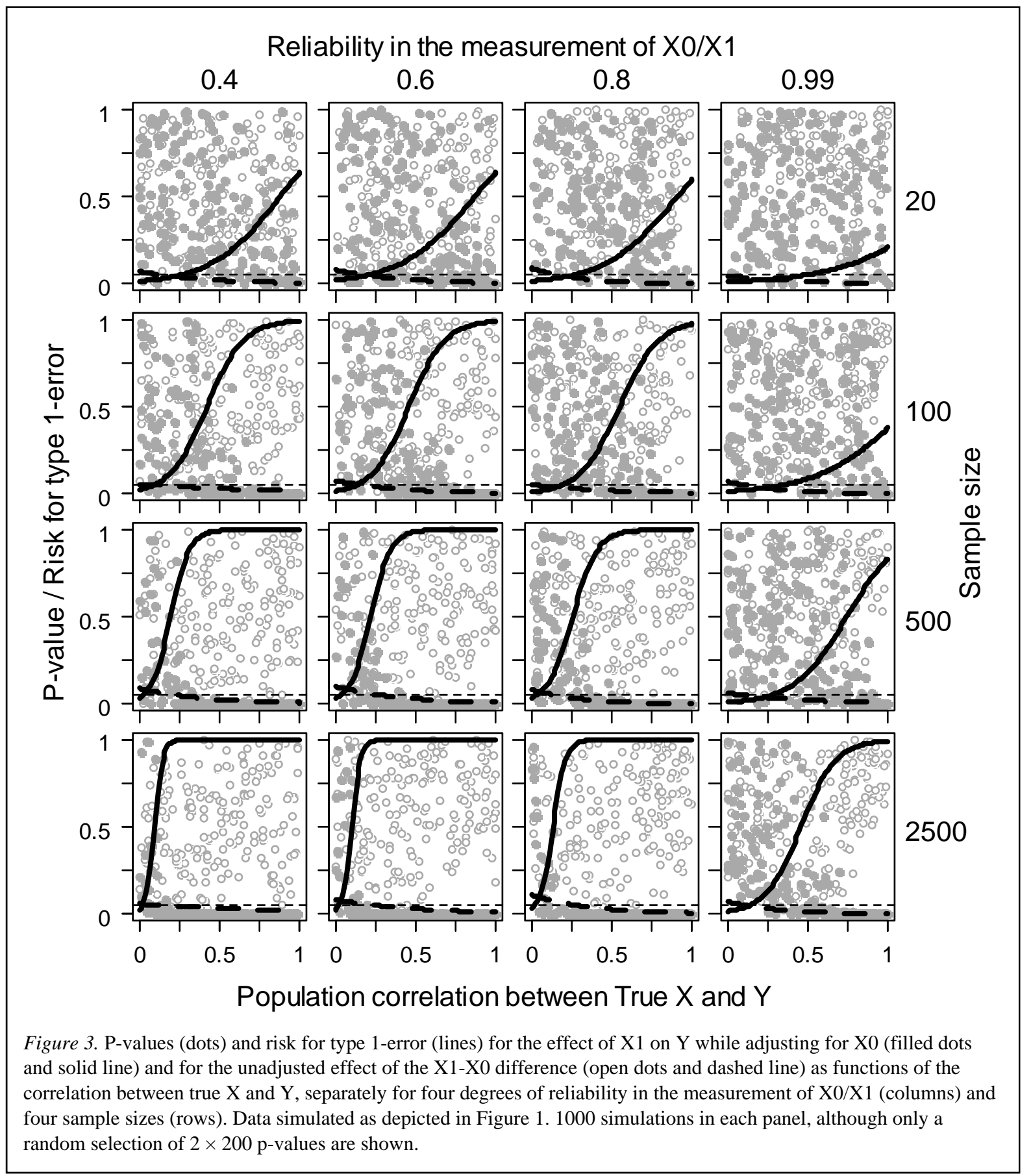


to have higher power, the recommendation should be unavoidable: Researchers should look at the unadjusted effect of the X1-X0 difference, rather than the effect of $\mathrm{X} 1$ while adjusting for $\mathrm{X} 0$, if wishing to study whether change in $\mathrm{X}$ is predictive of a certain outcome Y.

Somebody might want to think that these deficits with the adjusted effect can be avoided by using the $\mathrm{X} 1$-X0 difference, rather than $\mathrm{X} 1$, as a predictor while adjusting for $\mathrm{X} 0$. However, this would give exactly the same results, which is easy enough to see because:

if $\mathrm{Y}=\mathrm{B}_{0}+\mathrm{B}_{1} \times \mathrm{X} 0+\mathrm{B}_{2} \times(\mathrm{X} 1-\mathrm{X} 0)(E q .3)$ then $\mathrm{Y}=\mathrm{B}_{0}+\mathrm{B}_{1} \times \mathrm{X} 0+\mathrm{B}_{2} \times \mathrm{X} 1-\mathrm{B}_{2} \times \mathrm{X} 0$ (Eq. 4) and $\mathrm{Y}=\mathrm{B}_{0}+\left(\mathrm{B}_{1}-\mathrm{B}_{2}\right) \times \mathrm{X} 0+\mathrm{B}_{2} \times \mathrm{X} 1$ (Eq. 5)

So, if including $\mathrm{X} 1-\mathrm{X} 0$ rather than $\mathrm{X} 1$ as a predictor in the model, the coefficient for $\mathrm{X} 0$ will change from $\mathrm{B}_{1}-\mathrm{B}_{2}$ to $\mathrm{B}_{1}$ but the coefficient for $\mathrm{X} 1-\mathrm{X} 0$ will be exactly the same as the coefficient for $\mathrm{X} 1$ (Cain et al., 1992). Given the same value on $X 0$, the value on $\mathrm{X} 1$ and the X1-X0 difference are completely exchangeable.

One way to evaluate if published findings could be due to an association between true $\mathrm{X}$ and $\mathrm{Y}$ rather than between a true change in $\mathrm{X}$ and $\mathrm{Y}$ is by comparing presented regression coefficients. For

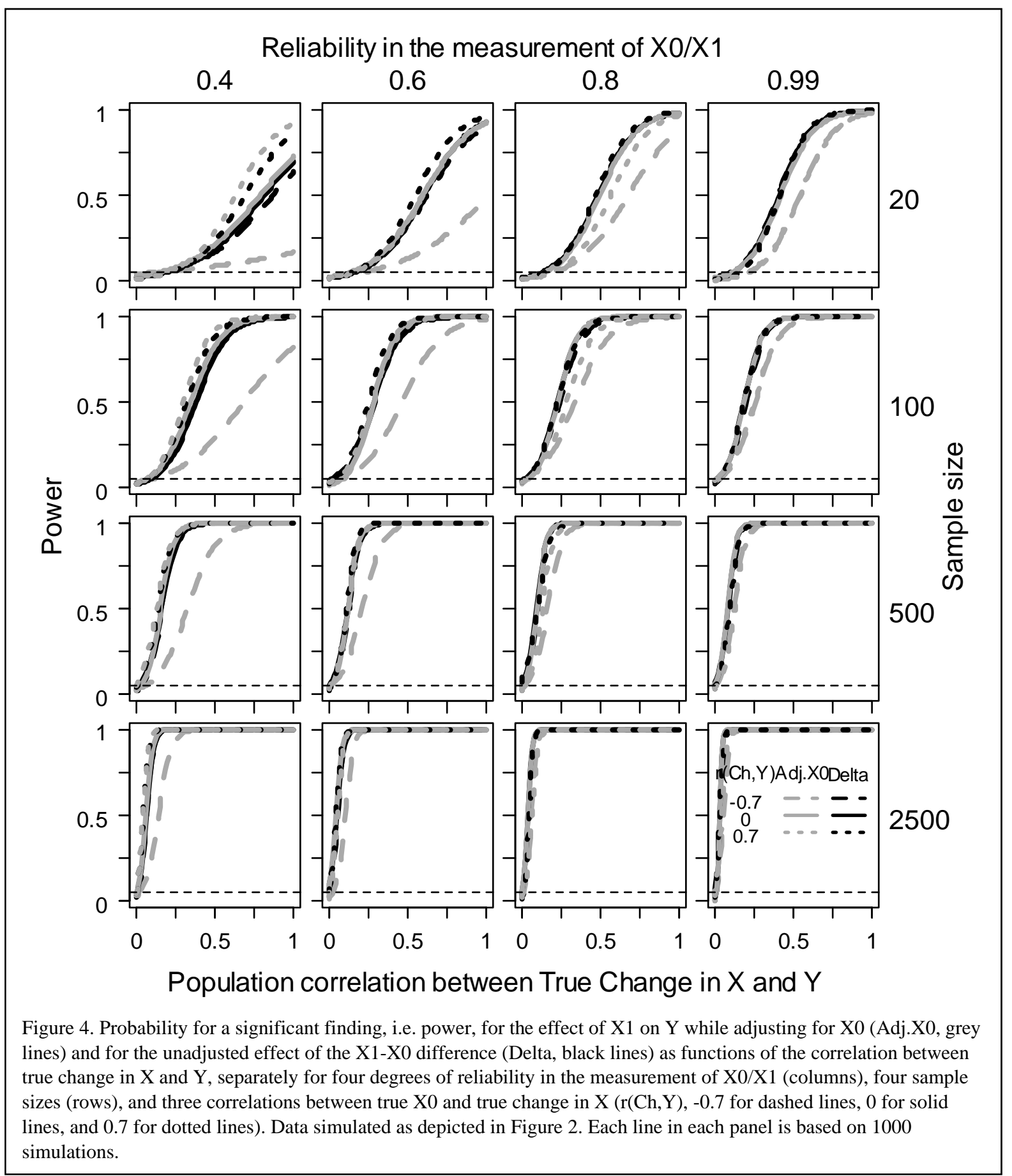




\begin{tabular}{|llcccc|}
\hline \multicolumn{7}{l}{ Table 1. Example of findings that do not seem to reflect a genuine effect of change. } \\
\hline \multicolumn{7}{l}{ Study } & Outcome & Predictor & $\mathrm{B}_{1}(\mathrm{X} 0)$ & $\mathrm{B}_{2}(\mathrm{X} 1-\mathrm{X} 0)$ & $\mathrm{B}_{1}<\mathrm{B}_{2}$ \\
\hline Menotti et al. (2004) & HR CVD deaths & SBP & 1.20 & 1.14 & No \\
Menotti et al. (2004) & HR All deaths & SBP & 1.15 & 1.11 & No \\
Parizadeh et al. (2017) & HR CVD & SBP & 1.35 & 1.15 & No \\
Parizadeh et al. (2017) & HR CVD & DBP & 1.42 & 1.24 & No \\
\hline Note: HR = Hazard Ratio, CVD = CardioVascular Disease, SBP = Systolic Blood Pressure, \\
DBP = Diastolic Blood Pressure.
\end{tabular}

example, if a significant positive $\mathrm{B}_{2}$-coefficient in Equation 3 above is to be taken to indicate that an increase in $\mathrm{X}$ is predictive of a high value on $\mathrm{Y}$, then a low value on $\mathrm{X} 0$ should be predictive of a high value on $\mathrm{Y}$ given the same value on $\mathrm{X} 1$ (given the same value on $\mathrm{X} 1$, the lower the value on $\mathrm{X} 0$, the more has $\mathrm{X}$ increased between the two time points). This means that we should see that $B_{1}-B_{2}<0$ (Equation 5), i.e. that $\mathrm{B}_{1}<\mathrm{B}_{2}$. Similarly, a significant negative $\mathrm{B}_{2}$-coefficient in Equation 3 indicates that a decrease in $\mathrm{X}$ is predictive of a high value on $\mathrm{Y}$ if $\mathrm{B}_{1}-\mathrm{B}_{2}>0$, i.e. if $\mathrm{B}_{1}>\mathrm{B}_{2}$. A failure to meet these predictions indicates that the found effect could be due to spurious associations (as depicted in Figure 1) rather than a genuine effect of change. This is the case for both studies on the effect of change in blood pressure mentioned in the introduction (Table 1).

\section{Conclusions}

If wishing to use the change between two time points on a certain variable (X1-X0) as a predictor of an outcome (Y), it is better not to adjust for the baseline value (X0) as this would increase the risk for type 1error while not giving any substantial increase in power.

\section{References}

Cain, K. C., Kronmal, R. A., \& Kosinski, A. S. (1992). Analysing the relationship between change in a risk factor and risk of disease. Statistics in Medicine, 11(6), 783-797. https://doi.org/10.1002/sim.4780110609

Cohen, J., Cohen, P., West, S. G., \& Aiken, L. S. (2003) Applied Multiple Regression/Correlation Analysis for the Behavioral Sciences. Mahwah, NJ: Lawrence Erlbaum Associates.

Eriksson, K., \& Häggström, O. (2014). Lord’s paradox in a continuous setting and a regression artifact in numerical cognition research. PLOS ONE, 9(4), e95949.

https://doi.org/10.1371/journal.pone.0095949

Menotti, A., Lanti, M., Kafatos, A., Nissinen, A., Dontas, A., Nedeljkovic, S., \& Kromhout, D. (2004). The role of a baseline casual blood pressure measurement and of blood pressure changes in middle age in prediction of cardiovascular and all-cause mortality occurring late in life: a cross-cultural comparison among the European cohorts of the Seven Countr. Journal of Hypertension, 22(9), 1683-1690. https://doi.org/10.1097/00004872-200409000-00011

Parizadeh, D., Ghahvehchian, H., Asgari, S., Momenan, A. A., Azizi, F., \& Hadaegh, F. (2017). The association between changes in blood pressure components and incident cardiovascular diseases. Blood Pressure, 26(6), 341-349. https://doi.org/10.1080/08037051.2017.1353882

R Core Team. (2018). R: A language and environment for statistical computing. R Foundation for Statistical Computing, Vienna, Austria. URL https://www.Rproject.org/.

Rosner, B. (1979). The analysis of longitudinal data in epidemiologic studies. Journal of Chronic Diseases, 32(1-2), 163-173. https://doi.org/10.1016/00219681(79)90045-6

Sorjonen, K., Melin, B., \& Ingre, M. (2019). Predicting the effect of a predictor when controlling for baseline. Educational and Psychological Measurement, 79(4), 688-698. https://doi.org/10.1177/0013164418822112 


\section{Appendix}

According to Figure 1, the expected correlations between $\mathrm{X} 0 / \mathrm{X} 1$ and $\mathrm{Y}$ are given by:

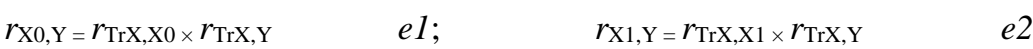

If we replace terms in Equation 1 (see Method section) with $e 1$ and $e 2$ we get:

$$
\beta_{X 1, Y, X 0}=\frac{r_{T r X, X 1} \times r_{T r X, Y}-r_{T r X, X 0} \times r_{T r X, Y} \times r_{X 0, X 1}}{1-r_{X 0, X 1}^{2}}
$$

We assume equally strong correlations between $\operatorname{Tr} \mathrm{X}$ and $\mathrm{X} 0 / \mathrm{X} 1$ and, therefore:

$$
r_{\operatorname{Tr} X, X 0}=r_{\operatorname{Tr} X, X 1}=\sqrt{r_{X 0, X 1}}
$$

If we replace terms in $e 3$ with $e 4$ we get:

$$
\beta_{X 1, Y . X 0}=\frac{\sqrt{r_{X 0, X 1}} \times r_{T r X, Y}-\sqrt{r_{X 0, X 1}} \times r_{T r X, Y} \times r_{X 0, X 1}}{1-r_{X 0, X 1}^{2}}
$$

e5 simplifies to:

$$
\beta_{X 1, Y . X 0}=\frac{\sqrt{r_{X 0, X 1}} \times r_{T r X, Y} \times\left(1-r_{X 0, X 1}\right)}{1-r_{X 0, X 1}^{2}}
$$

Both $e 1$ and $e 2$ can be used to calculate the expected correlation between $\operatorname{TrX}$ and Y. If we take the mean of these, and replace terms with $e 4$, we get:

$$
r_{T r X, Y}=\left(\frac{r_{X 0, Y}}{r_{T r X, X 0}}+\frac{r_{X 1, Y}}{r_{T r X, X 1}}\right) / 2=\frac{r_{X 0, Y}+r_{X 1, Y}}{2 \times \sqrt{r_{X 0, X 1}}}
$$

If we replace terms in $e 6$ with $e 7$ and expand the denominator we get:

$$
\beta_{X 1, Y . X 0}=\frac{\sqrt{r_{X 0, X 1}} \times\left(\frac{r_{X 0, Y}+r_{X 1, Y}}{2 \times \sqrt{r_{X 0, X 1}}}\right) \times\left(1-r_{X 0, X 1}\right)}{\left(1+r_{X 0, X 1}\right) \times\left(1-r_{X 0, X 1}\right)}
$$

If we multiply the nominator and the denominator by $2, e 8$ simplifies to:

$$
\beta_{X 1, Y . X 0}=\frac{r_{X 0, Y}+r_{X 1, Y}}{2 \times\left(1+r_{X 0, X 1}\right)}
$$

e9 gives the expected effect of $\mathrm{X} 1$ (or of the $\mathrm{X} 1-\mathrm{X} 0$ difference) on $\mathrm{Y}$ when adjusting for $\mathrm{X} 0$ in a situation with no true effect of change in $\mathrm{X}$ on $\mathrm{Y}$, as depicted in Figure 1. 